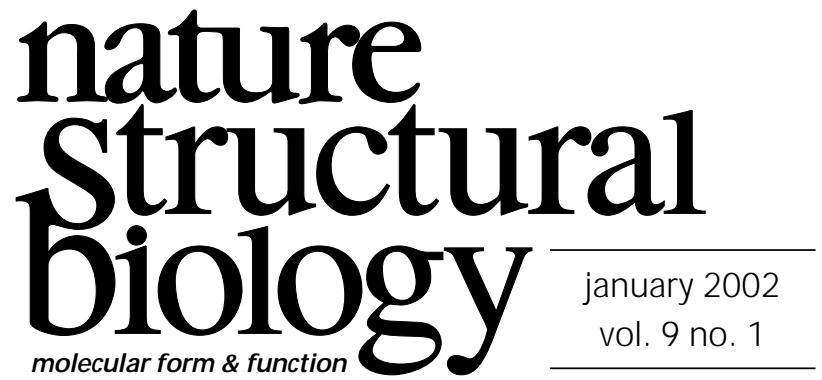

\title{
Designer drug, designed therapy
}

Cocaine is found in the leaves of a South American bush called Erythrocylon coca. For thousands of years, the Incas have known that chewing coca leaves elicits euphoric effects. In addition, cocaine has anesthetic properties, and its medicinal use dates as early as the $16^{\text {th }}$ century. Between the mid $19^{\text {th }}$ and early $20^{\text {th }}$ centuries, cocaine was an ingredient of many tonics and elixirs (including the early Coca Cola, which was originally marketed as a therapeutic beverage) that were developed to treat a variety of illnesses. Today, a compound that is related to cocaine - that is, novocaine - is still in use as a local anesthetic for dental surgeries.

While the use of cocaine as medication has been documented, its addictive stimulatory effects are much better known to the general public. Within minutes to hours after intake, the cocaine user usually feels energetic, talkative and mentally alert. However, the rush of euphoric feelings is short-lived. In trying to reach the 'high' induced by cocaine again and again, the user repeatedly ingests cocaine, often with higher and higher doses, eventually becoming addicted to the drug.

Cocaine became a very popular drug during the ' 80 s and early '90s. Although the fraction of the population using cocaine has decreased in recent years compared to 1985, the widespread abuse of this drug is still a serious problem. A 1999 survey conducted by the United Nations International Drug Control Programme (UNDCP) estimates that $\sim 13$ million people worldwide are current users of cocaine. Furthermore, the toxic effects of cocaine are cited as the most significant cause for drug-related visits to the emergency room during the first half of 2000 in the US. These data indicate that treatments for both cocaine addiction and overdose are urgently needed.

There are two possible approaches to designing treatments against the effects of cocaine. One is to understand the molecu- lar mechanisms of cocaine addiction and then block the actions of the drug. Much research effort has been devoted to this task, and accumulating evidence indicates that cocaine affects the release and reabsorption of several neurotransmitters, including dopamine, which is involved in stimulating the brain's reward system. The molecules responsible for transporting these neurotransmitters are therefore attractive targets for therapeutics.

A second way to stop the effect of cocaine is to prevent it from reaching its targets. For example, antibodies against cocaine could specifically bind to the drug in the blood and prevent it from crossing the blood-brain barrier. This could significantly reduce the amount of cocaine reaching the brain. In fact, a vaccine for cocaine based on this strategy has already been developed and is now in phase II clinical trials.

While antibody binding could be an effective way to reduce the level of cocaine in the brain, this may not help overdose patients; the drug concentration in the blood may simply exceed the binding capacity of the antibodies. In this case, enzymatic breakdown of cocaine into harmless metabolites would be an effective way to rapidly clear the drug. Based on this strategy, prototypical antibodies capable of hydrolyzing cocaine have been engineered. However, their catalytic efficiencies are not high enough to be effective overdose treatments. Understanding how naturally occurring enzymes degrade cocaine will provide valuable information to improve the efficiency of those catalytic antibodies. The structure of a cocaine esterase from a strain of bacteria that grows and thrives on cocaine as a food source, reported on page 17 of this issue, provides a starting point for designing the next generation of therapy. 